A theorist who puts thin coronae

In 0 stars (though some think it phoney)

Is ready to swear

That they really are there

on the gospel according to Tony.

SESSION 3

INFRARED AND RADIO DATA

Chairman: A. UNDERHILL

Introductory Speaker: M.J. BARLOW

1. J.M. VREUX and $Y$. ANDRILLAT: Near infrared spectra of o stars and related objects.

2. P. PERSI, M. FERRARI-TONIOLO and G. SPADA: Infrared observations of HDE $226868 /$ Cyg $X-1$ and HDE $245770 /$ A0 $535+26$.

3. A.R. HYLAND: Infrared excesses and mass loss: Implications for OB stars in Carina and the LMC.

4. P.R. SCHWARTZ: Radio emission from flows.

5. S. KWOK and C.R. PURTON: Radio observations of stellar mass loss.

6. D.C. MORTON and A.E. WRIGHT: Radio emission from hot stars at two centimeters. 


\title{
RADIO AND INFRARED EMISSION BY 0-TYPE AND RELATED STARS
}

\author{
M. J. Barlow
}

Anglo-Australian Observatory, P.0. Box 296, Epping, N.S.W. 2121, Australia.

\section{INTRODUCTION}

In addition to 0-type stars, this review will discuss the radio and infrared properties of $B$ supergiants and Wolf-Rayet stars, since it is generally accepted that these objects represent later stages in the evolution of 0 stars. The radio properties of X-ray binaries which exhibit non-thermal emission will not be discussed. Hjellming (1977) gives an excellent review of the radio properties of close binaries, including $\mathrm{X}$-ray binaries.

\section{RADIO OBSERVATIONS OF MASS LOSS}

Johnson (1971) recognised that Wolf-Rayet stars, due to their very large mass loss rates, offered the best chance of a radio detection. of a stellar wind. Consequently, he observed nine Wolf-Rayet stars at 2.7 $\mathrm{GHz}$, but due to the low receiver sensitivities and large beamwidths then current, no detections were obtained. Balick (1972) set upper limits of between 20 and $50 \mathrm{mJy}$ at 2.7 and $8.1 \mathrm{GHz}$ for 320 , B and Wolf-Rayet stars. The first definite detection of mass loss from an early-type star was by Wendker, Baars and Altenhoff (1973), who detected P Cygni at 5 and $10.7 \mathrm{GHz}$ and found a spectral index of $0.7 \pm 0.3$ (if the flux $\mathrm{S}_{V}$ at a frequency $\nu$ is proportional to $\nu^{\alpha}$, then $\alpha$ is defined as the spectral index). Wendker et al. (1975) found a source of $3.5 \pm 1.5 \mathrm{mJy}$ at $5 \mathrm{GHz}$ coincident with the $\overline{\mathrm{WN}} 6$ Wolf-Rayet star HD 192163. Seaquist (1976) detected the Wolf-Rayet binary $\gamma$ Vel at three frequencies between 5 and $8.87 \mathrm{GHz}$ and found a spectral index of $\alpha=0.64 \pm 0.32$. The lists of $10.7 \mathrm{GHz}$ upper 1imits given by Altenhoff et al. (1976) and by Woodsworth and Hughes (1977) include several 0 and B stars. Florkowski and Gottesman (1977) detected the WC7+05 Wolf-Rayet system HD 193793 at 2.7 and $8.1 \mathrm{GHz}$. This star will be discussed in more detail in section 4. Schwartz and Spencer (1977) obtained a $2 \sigma$ detection of P Cygni at $90 \mathrm{GHz}$, confirming that the spectral index of 0.7 between 5 and $10 \mathrm{GHz}$ extended to higher frequencies. Morton and Wright (1978, and this 
Symposium) detected Zeta Pup at $14.7 \mathrm{GHz}$ and also measured the flux from $\gamma$ Vel at the same frequency. Schwartz (this Symposium) has detected the Oe star HD 60848 at $2.7 \mathrm{GHz}$.

Seaquist and Gregory (1973) have shown that a spherically symmetric plasma density distribution, which is radially decreasing outward, leads to a spectral index intermediate between $\alpha=-0.1$ and +2 . The latter values correspond to optically thin and thick free-free emission. Panagia and Felli (1975) and Wright and Barlow (1975) showed that a density distribution $\mathrm{N} \propto \mathrm{r}^{-\mathrm{B}}$ gives rise to a radio spectral index

$$
\alpha=\frac{4 \beta-6.2}{2 \beta-1}
$$

They solved the radiative transfer equation for the case of $\beta=2$ (constant expansion velocity) in order to derive expressions for the total flux at a given frequency and the mass loss rate corresponding to an observed flux (Thorne (1975) independently rederived the same results not long afterwards). Thus, for an isothermal wind it was found that

$$
\begin{array}{r}
s_{v} \propto\left(\frac{\dot{M}}{v_{\infty}}\right)^{4 / 3} \frac{v^{2 / 3} g^{2 / 3}}{D^{2}} \\
\text { i.e. } \frac{\dot{M}}{v_{\infty}} \propto s_{v}{ }^{3 / 4} D^{3 / 2} v^{-\frac{1}{2}} g^{-\frac{1}{2}}
\end{array}
$$

$\dot{M}$ is the mass loss rate, $v_{\infty}$ is the terminal velocity of the wind and $D$ is the distance of the star. $S_{\nu}$ is the flux received at frequency $\nu$ and $\mathrm{g}$ is the Gaunt factor. It is the weak frequency dependence of the Gaunt factor in the radio which flattens the spectral index from $\alpha=2 / 3$ to $\alpha=0.6$. Relationship (2) shows that for a given mass loss rate a lower terminal velocity will lead to a larger radio flux and consequently a better chance of detection.

Relationships (2) and (3) are valid for the radio spectral region only if the wind has reached its terminal velocity in the radio-emitting volume. This can easily be shown to hold, since the characteristic radio-emitting radius, obtained from equation (11) of Wright and Barlow (1975) is typically between several tens to hundreds of stellar radii, at which point the wind will have reached terminal velocity for any plausible acceleration law. If $n \propto r^{-2}$ then $S_{V}$ is independent of the wind temperature, except weakly through the Gaunt factor. However, if the wind temperature varies systematically with radius, then this Gaunt factor dependence on temperature can cause the spectral index to be modified from the value of $\alpha=0.6$ appropriate for an isothermal wind to values up to $\alpha=0.83$, as shown by Thorne (1975) and by Chiuderi and Torricelli Ciamponi (1978). Both these treatments give formulae for the spectral index for the case of a wind undergoing cooling by adiabatic expansion. The expression for $\alpha$ from the latter paper is 


$$
\alpha=\frac{1.5-0.6 \gamma}{2.85-1.35 \gamma}
$$

Where $\gamma$ is the adiabatic exponent of the gas. Thus, $\alpha$ can range from the standard isothermal value of $\alpha=0.6$ for $\gamma=1$, up to $\alpha=0.83$ for $\gamma=5 / 3$.

Thorne (1975) has discussed the criteria for adiabatic cooling to be important in a wind, pointing out that until adiabatic cooling is significant, the electron temperature and ionization state in the flow will remain constant, since they depend only on the Tarter-TuckerSalpeter parameter $\mathrm{L} / \rho \mathrm{r}^{2}$, which is a constant for regions where $\mathrm{v}=\mathrm{v}_{\infty}$. The gas will start to cool when the adiabatic cooling rate $\mathrm{E}_{\mathrm{ad}}=$ $-2 \mathrm{kT} v / \mathrm{r}$ is comparable to the radiative cooling rate due to recombination, bremsstrahlung and collisional excitation, $\dot{\mathrm{E}}_{\text {rad }}=\mathrm{Ln} \mathrm{n}_{\mathrm{H}}$. From the work of Cox and Caltabuit (1971) and Raymond, Cox and Smith (1976) I can crudely but adequately set $\mathrm{L}=5 \times 10^{-27} \mathrm{~T}$ erg $\mathrm{cm}^{3} \mathrm{~s}^{-1}$, for electron temperatures $\mathrm{T}$ in the range $10^{4} \mathrm{~K}-2 \times 18^{5} \mathrm{~K}$ (this is strictly only valid for collisionally ionized plasmas at a temperature $T$, but a glance at Figure 3 of Cox and Daltabuit (1971) shows that the most important permitted coolant ions are those known to be present in 0 star winds). Setting $\dot{E}_{\text {ad }}=\dot{E}_{\text {rad }}$, we obtain the radius $r_{c o o l}$ at which
adiabatic cooling becomes

$$
\mathrm{r}_{\text {cool }}=4.3 \times 10^{15} \frac{\dot{\mathrm{M}}_{-6}}{\mathrm{v}_{8}^{2}} \mathrm{~cm}
$$

where $\dot{M}$ is the mass loss rate in units of $10^{-6} \mathrm{M}_{\odot} \mathrm{yr}^{-1}$ and $\mathrm{v}_{8}$ is the termina $\overline{1}^{6}$ velocity in units of $10^{8} \mathrm{~cm} \mathrm{~s}^{-1}$. Adopting $\mathrm{M}^{\circ}=7$ and $v_{8}=2.66$ from Lamers and Morton (1976) and Morton and Wright (1978) we obtain $r=4.3 \times 10^{15} \mathrm{~cm}$ for Zeta Pup. This can be compared with the characteristic radius for $5 \mathrm{GHz}$ radio emission, equal to $7.6 \times 10^{13} \mathrm{~cm}$ for Zeta Pup, using equation (11) of Wright and Barlow (1975). For all likely $O B$ star mass loss rates, the adiabatic cooling radius is always much larger than the characteristic radio emitting radius. For WolfRayet stars the radiative cooling rate will be slightly different from the cosmic abundances rate used above, but the conclusions remain the same. Since no mechanical energy deposition is expected at the large characteristic radio emitting radii of $O B$ and WR stars ( 30-150 stellar radii), the isothermal wind temperatures in these regions should be $\sim 0.9 \mathrm{~T}_{\text {eff }}$ (Klein and Castor 1978), where $\mathrm{T}_{\text {eff }}$ is the effective tem-
perature of the star.

Thus it is predicted that early-type stars undergoing mass loss should always have isothermal winds in their radio emitting regions, giving rise to a spectral index of 0.6 . The only early-type star with radio detections of high enough signal-to-noise for detailed checking of this prediction is $\gamma$ Vel. Seaquist (1976) found $36 \pm 5 \mathrm{mJy}$ at $5 \mathrm{GHz}$ and $52 \pm 6 \mathrm{mJy}$ at $8.87 \mathrm{GHz}$ and Morton and Wright (this Symposium) found $69 \pm 5 \mathrm{mJy}$ at $14.7 \mathrm{GHz}$. Thus, spectral indices of $0.55 \pm 0.25$ and $0.64 \pm 0.26$ are found between 14.7 and $8.87 \mathrm{GHz}$ and 8.87 and $5 \mathrm{GHz}$, 
respectively, and over the largest frequency baseline of 14.7 to $5 \mathrm{GHz}$ a spectral index of $0.60 \pm 0.14$ is found, confirming the isothermal mode 1 prdiction.

01non (1975) has derived the radio spectral indices corresponding to power-law, Caussian and inner-truncated density distributions, while Marsh (1975) has considered the case of an outer-truncated density distribution. Panagia and Felli (1975) demonstrated that the radius at which a stellar wind interacts with the interstellar medium is much larger than the characteristic radio-emitting radius. Therefore, in the absence of variability, a simple $r^{-2}$ density distribution and thus a 0.6 spectral index should always apply to the radio emission from $O B$ and WR stars.

\section{INFRARED OBSERVATIONS OF MASS LOSS}

\subsection{OB stars}

Infrared observations of early-type stars are scattered throughout the literature and are thus less easy to summarise than the radio data. I will accordingly concentrate mainly on the work which has been concerned with mass loss aspects.

Woolf, Stein and Strittmatter (1970) interpreted the 2-10 $\mathrm{mm}$ excesses of a number of early-type stars (mainly classical Be stars, apart from $P$ Cyg) as due to free-free emission from hot ionised shells surrounding the stars. Gehrz, Hackwell and Jones (1974) obtained 2-20 $\mu$ photometry of a much larger sample of Be stars, and in addition included a number of early-type Supergiants such as P Cyg. The analysis used in the above papers invoked a constant density shell of finite radius surrounding each star, giving optically thin and thick free-free radiation shortward and longward of a critical wavelength $\lambda_{c}{ }^{\circ}{ }_{0}$. This wavelength $\lambda_{c}$ was deduced for each star as the intersection of $v^{0 \cdot 1}$ and $\nu^{-2}$ power laws fitted to the excess flux distributions. While perhaps valid for 'shell' Be stars, this approach would clearly not be applicable to stars undergoing continuous mass loss, since then a range of densities is present around each star, giving an infrared spectral index intermediate between -0.1 and +2 , as discussed in the previous section.

The 2-20um free-free emission from mass loss outflows originates from regions close to the stellar surface (typically between 1 and 5 stellar radii). Consequently we may expect that the density distribution in this region will be steeper than $\mathrm{r}^{-2}$ since the wind will be undergoing acceleration up to its terminal velocity. Additionally, the temperature $T$ of the wind may vary with radius, due to mechanical energy input or ther reasons. Hartmann and Cassinelli (1977) have shown that if $\mathrm{N} \propto \mathrm{r}^{-B^{2}}$ and $\mathrm{T} \propto \mathrm{r}^{-\mathrm{m}}$, the (frequency) spectral index $\alpha$ is given.

$$
\alpha=\frac{4 \beta-m-6}{2 \beta-\frac{3}{2} m-1}
$$


For $m=0$ (isothermal) this reduces to the standard case (1) (ignoring the Gaunt factor), while for $\beta=2$ (constant expansion velocity) $\alpha=2 / 3$, independent of temperature variations (again ignoring the Gaunt factor effects discussed in the previous section).

Thus, if temperature gradients are present in the region of acceleration of a wind, the interpretation of the infrared data will be somewhat complicated. However, judicious model fitting can be used to set some limits on temperature gradients in cases where the mass loss rate is known from radio data.

The infrared spectrum of $\mathrm{P}$ Cyg $\left(\mathrm{B}_{1} \mathrm{I}^{+}\right)$has been discussed in detail by Barlow and Cohen (1977). The optical and ultraviolet spectra of this star show only low-excitation emission lines and so the assumption of an isothermal wind of temperature $\mathrm{T} \sim \mathrm{T}$ ff would appear to be justifiable. Barlow and Cohen showed that the observed infrared fluxes were too large and the spectral index between the infrared and radio regions too steep, to be consistent with a constant outflow velocity in the infraredemitting region. An extended acceleration zone was required in order to increase these quantities to their observed values. The decelerating wind model of Kuan and Kuhi (1975) was shown to be inconsistent with the observed infrared flux distribution. The derived velocity law was significantly less steep than the original theoretical velocity law of Castor, Abbott and Klein (1975). Barlow and Cohen were also able to show that, for a given velocity law, density clumping in the wind would lead to larger mass loss rates being derived from $\mathrm{H} \alpha$ fluxes than from infrared fluxes. Since the observed fluxes from a number of stars do not give this result, it would appear that significant density clumping does not occur in mass outflows from 0 stars.

The application by Barlow and Cohen of the empirical P Cyg velocity law to the infrared excesses of other $B$ supergiants and a few 0 stars allowed their mass loss rates to be determined. The run of $\varepsilon$ ( $\varepsilon=$ mass loss rate $\dot{M}$, divided by $\mathrm{L} / \mathrm{v}_{\infty} \mathrm{c}$, the upper limit for mass 1 oss by single scattering radiation pressure) versus effective temperature, and the dependence of $\dot{M}$ on stellar luminosity $L\left(\dot{M} \propto L^{1 \cdot 15}\right)$ were both consistent with the radiation pressure mechanism of Lucy and Solomon (1970), as developed by Castor et al. (1975).

It is obvious that additional velocity law determinations are needed for stars more 'normal' than the extreme supergiant $P$ Cyg. Work underway on the infrared flux distribution of Zeta Pup indicates that application of a more appropriate 'faster' velocity law to 0 star infrared excesses should not increase the derived mass loss rates by more than about a factor of two over the values determined using the $P$ Cyg velocity law.

Cassinelli and Hartmann (1977) have shown that an extended coronal region $\left(\triangle \mathrm{R} / \mathrm{R}=0.3, \mathrm{~T} \sim 2 \times 10^{6} \mathrm{~K}\right)$ at the base of an 0 star wind would give rise to a rather flat infrared flux distribution with a broad bump at $\lambda \sim 30 \mu \mathrm{m}$. The observed 2.2-20 $\mathrm{m}$ spectrum of Zeta Pup rules out this 
model since the excess flux distribution is rather steep $(\alpha \sim 1)$. The infrared observational data do not however rule out the thin corona model $(\triangle R / R<0.1)$ of Cassinel1i, 0lson and Stalio (1978) and Cassinelli and 01son (1978).

Barnes, Lambert and Potter (1974) obtained a high resolution 0.9$1.7 \mu \mathrm{m}$ spectrum of Zeta Pup which showed an absorption line spectrum apart from very weak He II emission at $10124 \AA$ (Pickering $\alpha$ ). Emission lines are expected to make a negligible contribution to the infrared excesses of $O B$ stars. No evidence has ever been found for infrared thermal dust emission from $O B$ star winds. Some 0 stars are of course embedded in $\mathrm{H}$ II regions which are infrared sources.

\subsection{Wolf-Rayet stars}

Allen, Harvey and Swings (1972) observed a large sample of WolfRayet stars at 1.6 and $2.2 \mu \mathrm{m}$. They found that the infrared excesses from WN and early WC stars were consistent with free-free emission, whereas the WC9 stars had excesses which were too large to be explained by this mechanism. By obtaining photometry to longer wavelengths (3.5$10 \mu \mathrm{m})$, Allen et al. showed that the WC9 excesses could be fitted almost perfectly by single-temperature black-body curves of temperature $\mathrm{T} \sim$ $1000 \mathrm{~K}$, attributable to dust emission. Further long-wavelength photometry and more detailed dust shell models for the WC9 stars can be found in Gehrz and Hackwell (1974) and Cohen, Barlow and Kuhi (1975). The lack of strong spectral emission features together with the known carbonrichness of WC stars strongly suggests that the dust is a condensate of carbon, such as graphite. Thomas, Robinson and Hyland (1976) obtained intermediate bandwidth photometry $(\Delta \lambda \sim 2 \mu \mathrm{m})$ in the $8-13 \mu \mathrm{m}$ region for the WC9 star Ve2-45. They found evidence for a weak emission feature at $\lambda \sim 10-12 \mu \mathrm{m}$, possibly due to emission by SiC particles. The SiC emission feature is known to be present in the infrared spectra of many carbon stars whose infrared excesses are thought to be dominated by carbon particle emission.

In addition to the above WC9 stars, CRL 2104 (WC8) and CRL 2179 (WC9), have been found to show strong circumstellar dust emission (Cohen and Kuhi 1976, Allen et a1. 1977). Another WC8 star, CV Ser, probably has a dust she11, the infrared excess being rather large to be explained by free-free emission (Cohen et al. 1975). The same probably holds for the $3 \mu \mathrm{m}$ excess of the WC7+Be star HD 192641 (Cohen and Vogel 1978). The WC8+09I star $\gamma$ Vel shows no dust emission in the infrared (Allen and Porter 1973; Seaquist 1976). Thus all northern (Allen et al. 1972, Gehrz and Hackwell 1974, Cohen et al 1975) and southern (Allen and Porter 1973; Cohen 1975) WC9 stars show circumstellar dust emission, whereas some WC7 and WC8 stars show dust emission and some do not.

The analysis by Hackwe11, Gehrz and Smith (1974) and Cohen et al. (1975) of the free-free emission from WN and early-WC stars was done in the framework of a constant density, finite radius, shell model. A more detailed analysis in terms of an expanding wind model is therefore 
needed. The 10 $\mu \mathrm{m}-(5-15) \mathrm{GHz}$ spectral indices of $\gamma$ Vel (WC8+09I) and HD 192163 (WN6) are 0,71 and 0.67 , respectively, whereas the spectral indices of $\mathrm{P}$ Cyg $\left(\mathrm{B} 1 \mathrm{I}^{+}\right.$) and Zeta Pup (04f) between the same spectral regions are 0.76 and 0.74 respectively. The less steep spectral indices of the Wolf-Rayet stars can be interpreted as evidence that accelerative effects are a little less important in distorting the spectral index from the constant velocity value. Thus the $10 \mu \mathrm{m}$ radiation from WolfRayets probably originates from a region closer to the wind terminal velocity than is the case for the OB stars.

Although detailed models are needed for the infrared emitting regions, one may use $10 \mu \mathrm{m}$ fluxes, combined with the mean infrared-radio spectral index from $\gamma$ Vel and HD 192163, to derive approximate mass loss rates for a variety of Wolf-Rayet stars. The mass loss rates obtained range from $2 \times 10^{-5} \mathrm{M} / \mathrm{yr}$ for some WN5 stars up to $1 \times 10^{-4} \mathrm{M} / \mathrm{yr}$ for WN7, WN8 and WC8 stars. These mass loss rates are larger by factors of 5 to 40 than the upper limit for mass loss by single-scattering radiation pressure, $\mathrm{L} / \mathrm{v}_{\infty} \mathrm{c}$, in contrast to the $O B$ stars, which have mass loss rates less than this upper limit. Thus the mass loss phenomenon in Wolf-Rayet stars is orders of magnitude more extreme than 0 star mass loss.

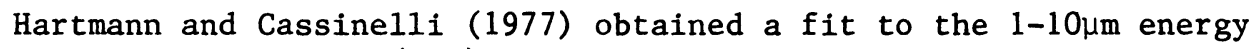
distribution of HD 50896 (WN5) by using a density distribution which incorporated an extended deceleration zone out to several stellar radii, followed by a zone of rapid acceleration to terminal velocity. Hartmann (1978) modeled the density structure of the wind of the WN5 component of V444 Cyg by using 2.2 and $3.5 \mu \mathrm{m}$ photometry of the eclipse of the system and obtained results consistent with the decelerating wind hypothesis. Since a decelerating wind is a surprising result physically and is of intrinsic interest if confirmed, more work should be carried out on the structure of Wolf-Rayet winds, using data from all spectral regions.

Barnes et al. (1974) obtained high resolution spectra of $\gamma$ Vel between 0.9 and $1.7 \mu \mathrm{m}$, at a resolution of $\sim 4 \times 10^{3}$. Their spectra show several strong emission lines of He I, He II, C III and C IV. Cohen and

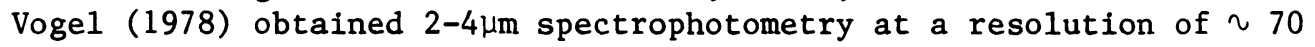
for several late WC stars and one WN6 star. Their data show mainly emission lines of He I and He II in this spectral region, although an unidentified emission feature at $3.29 \mu \mathrm{m}$ is often present. Those stars known to have dust shells exhibit diluted gaseous emission lines, some having dust emission so strong that the lines appear to be completely washed out, since no trace of them is apparent. The spectrophotometry of Cohen and Vogel (1978) shows that line emission is not a significant contaminant of WR free-free fluxes deduced using standard broad-band 2.2 and $3.5 \mu \mathrm{m}$ filters.

\section{INFRARED AND RADIO VARIABILITY}

Hackwel1 et al. (1976) presented evidence for a systematic decrease between 1970 and 1975 of the infrared fluxes of the Wolf-Rayet stars 
HD 193793 ( $W C 7+05$ ) and HD 192641 (WC7+Be), the changes being most pronounced in the former star. No other Wolf-Rayet star which they monitored showed evidence for variability. They interpreted their results for these two stars in terms of a steadily decreasing mass loss rate with time. Florkowski and Gottesman (1977) detected HD 193793 in October 1975 at 2.7 and $8.1 \mathrm{GHz}$. The spectral index of -0.19 which they found was inconsistent with an $\mathrm{r}^{-2}$ density distribution, corresponding instead to optically thin free-free emission. Florkowski has since reported (Wood 1977) that observations of HD 193793 in March 1977 revealed that the radio flux had declined by at least a factor of five since the 1975 measurements and that the spectral index was at least 0.6. Hackwell has also reported (Wood 1977) that infrared observations in May 1977 showed the infrared flux of HD 193793 to have increased and reached almost its 1970 value. The infrared magnitudes of HD 193793 obtained by Williams et al. (1977) in September 1977 were brighter by a full magnitude at $K$ and $\mathrm{L}$ than the 1970 data of Hackwell et a1. (1976). Williams et al. suggested that a dust shell had formed. Thus HD 193793 has exhibited complex variability in both the infrared and radio regions. In this regard it is interesting that Davies et al. (1967) claimed a detection of HD 193793 at a $2.7 \mathrm{GHz}$ flux level of $320 \pm 90 \mathrm{mJy}$, although the 15 arcmin beamwidth used by these authors makes confusion more of a problem. The flat radio spectral index found by Florkowski and Gottesman (1977) in October 1975 might be interpretable as a shell ejection event, the shell having become optically thin by the time it had travelled out to the radio emitting region (travel time $\sim 1$ month). Continued monitoring of HD 193793 at all wavelengths is essential. Morton and Wright (this Symposium) have found no evidence for any radio variability of the WC8+09I star $\gamma$ Vel.

Infrared photometry which I obtained for Xi Per (07.5III) in August 1976 showed this star to be systematically fainter at all wavelengths than the 1972-3 photometry of Gehrz et al. (1974), the difference being greater than 1 magnitude at $10 \mu \mathrm{m}$. Since the infrared excess in 1976 was similar to that of other 0 stars of the same spectral type, it would appear that Xi Per was undergoing an outburst when observed by Gehrz et al. Some evidence for this exists also in the radio data of Bohnenstengel and Wendker (1976), who found a radio component at $2.7 \mathrm{GHz}$ coincident with Xi Per, in observations made during the last quarter of 1972 . Later observations which they made apparently falled to show the same radio component. Thus a supposedly ordinary 07.5 III star shows evidence for large variations in its mass loss rate. The importance and frequency of such mass loss events needs to be fully explored for a range of luminosity and spectral types.

\section{FUTURE PROSPECTS}

The radio region is probably the best spectral domain for determining accurate mass loss rates for individual stars. Once the radio flux at a given frequency has been measured, only the terminal velocity 
of the wind (obtainable from ultraviolet spectra) and the distance of the star are required in order to obtain the mass loss rate. Once $\dot{M}$ is known, model fitting to the infrared flux distribution, in combination with Balmer line profile fitting, probably provides the best method of determining the velocity law for an individual stellar wind. When $\dot{M}$ and the velocity law have been determined using these methods, the detailed physics of the wind can be explored using ultraviolet spectra.

Only a small number of early-type stars are detectable by the present generation of radio-telescopes. However, this situation will be completely changed with the completion of the Very Large Array (VLA) and the proposed Australian Synthesis Telescope (AST). When complete, the VLA is expected to be able to detect $(3 \sigma)$ fluxes as small as 10 microJanskys $(\mu \mathrm{Jy})$ at $5 \mathrm{GHz}$. With this sensitivity a WC8 star like $\gamma$ Vel will be detectable to a distance of $30 \mathrm{kpc}$, an extreme B supergiant such as P Cyg will be detectable to $54 \mathrm{kpc}$, an $04 \mathrm{f}$ star such as Zeta Pup will be detectable to $10 \mathrm{kpc}$ and a main-sequence 08 star would be detectable at distances up to $1.6 \mathrm{kpc}$. The proposed AST is projected to be able to detect $80 \mu \mathrm{Jy}$ at $10 \mathrm{GHz}$, which will allow detections of the objects listed above to distances up to $60 \%$ as far as those appropriate for the VLA. Thus the AST should be able to detect $S$ Doradus and a few other extreme early-type supergiants in the Magellanic Clouds. Between them the VLA and AST will be able to detect every Wolf-Rayet star in the Galaxy (although some on the far side of the Galaxy may be optically invisible).

The proposed cryogenically cooled $1-\mathrm{m}$ telescope to be flown in the Space Shuttle (Gillett 1977) should be able to detect, at the $3 \sigma$ level is one hour's integration, fluxes as small as $4 \mathrm{mJy}$ at $100 \mu \mathrm{m}$, allowing a star such as Zeta Pup to be detected out to $3.3 \mathrm{kpc}$. This sytem should be factors of 7 and 40 more sensitive at $30 \mu \mathrm{m}$ and $10 \mu \mathrm{m}$ compared to $100 \mu \mathrm{m}$, although at the shorter wavelengths acceleration of the wind close to the star will affect the spectral index.

Another possible application of radio interferometers is to the determination of wind temperatures, since the characteristic radioemitting radius is proportional to $\mathrm{T}^{-\frac{1}{2}}$. However, even the $35 \mathrm{~km}$ baseline of the VLA will probably be inadequate to resolve the radio emission from most winds, e.g. the $5 \mathrm{GHz}$ angular diameter of $\mathrm{P}$ Cyg is expected to be $\sim 0.16$ arcsec, compared to a VLA resolution of $\sim 0.3$ arcsec at that frequency. Going to higher frequencies does not help much, since although the angular resolution is proportional to $\nu^{-1}$, the radio-emitfing radius of a wind is proportional to $v^{-2 / 3}$, giving only a factor of $v$ advantage. Thus much longer baselines will be required for wind temperature determinations. Systems such as the Jodrell Bank Multi-Telescope Radio-1inked interferometer with baselines of hundreds of $\mathrm{km}$ appear best suited to this application. 


\section{REFERENCES}

Allen, D.A., Harvey, P.M. \& Swings, J.P. 1972, Astr. Astrophys., $20,333$.

Allen, D.A., Hyland, A.R., Longmore, A.J., Caswell, J.L., Goss, W.M., \& Haynes, R.F. 1977, Astrophys. J., 217, 108.

Allen, D.A. \& Porter, F.C. 1973, Astr. Astrophys., 22, 159.

Altenhoff, W.J., Braes, L.L.E., Olnon, F.M. \& Wendker, H.J. 1976, Astr. Astrophys., 46, 11 .

Balick, B. 1972, Astrophys. Lett., 12, 21.

Barlow, M.J. \& Cohen, M. 1977, Astrophys. J., 213, 737.

Barnes, T.G., Lambert, D.L. \& Potter, A.E. 1974, Astrophys. J., 187,73 .

Bohnenstenge1, H.-D. \& Wendker, H.J. 1976, Astr. Astrophys., 52, 23.

Cassinel1i, J.P. \& Hartmann, L. 1977, Astrophys. J., 212, 488.

Cassine11i, J.P. \& 01son, G.L. 1978, Astrophys. J., in press.

Cassinel1i, J.P., 01son, G.L. \& Stalio, R. 1978, Astrophys. J., 220, 573.

Castor, J.I., Abbott, D.C. \& Klein, R.I. 1975, Astrophys. J., 195, 157 .

Chiuderi, C. \& Torricelli Ciamponi, G. 1978, preprint.

Cohen, M. 1975, Mon. Not. R. astr. Soc., 173, 489.

Cohen, M., Barlow, M.J. \& Kuhi, L.V. 1975, Astr. Astrophys., 40, 291.

Cohen, M. \& Kuhi, L.V. 1976, Publ. astr. Soc. Pacific, $88,535$.

Cohen, M. \& Vogel, S.N. 1978, Mon. Not. R. astr. Soc., in press.

Cox, D.P. \& Daltabuit, E. 1971, Astrophys. J., 167, 113.

Davies, J.G. Ferriday, R.J., Haslam, C.G.T., Moran, M. \& Thomasson, P. 1967, Mon. Not. R. astr. Soc., 135, 139.

Florkowski, D.R. \& Gottesman, S.T. $1 \overline{977}$, Mon. Not. R. astr. Soc., 179,105 .

Gehrz, R.D. \& Hackwe11, J.A. 1974, Astrophys. J., 194, 619.

Gehrz, R.D., Hackwe11, J.A. \& Jones, T.W. 1974, Astrophys. J., $191,675$.

Gillett, F.C. 1977, in 'Infrared and Submillimetre Astronomy', ed., G.G. Fazio (D. Reidel), p.195.

Hackwe11, J.A., Gehrz, R.D. \& Smith, J.R. 1974, Astrophys. J., 192, 383.

Hackwe11, J.A., Gehrz, R.D., Smith, J.R. \& Strecker, D.W. 1976, Astrophys. J., 210, 137 .

Hartmann, L. 1978, Astrophys. J., 221, 193.

Hartmann, L. \& Cassinel1i, J.P. 1977, Astrophys. J., 215, 155.

Hjellming, R.M. 1977, Proc. IAU Colloquium No.42, eds. R. Kippenhahn, J. Rahe, W. Strohmeier, p. 279.

Johnson, H.M., Proc. IAU Symp. No.49, eds., M.K.V. Bappu, J. Sahade (D. Reidel), p.42.

Klein, R. I. \& Castor, J.I. 1978, Astrophys. J., 220, 902.

Kuan, P. \& Kuhi, L.V. 1975, Astrophys, J., 199, 148.

Lamers, H.G.J.L.M. \& Morton, D.C. 1976, Astrophys. J. Suppl., 32, 715.

Lucy, L.B. \& Solomon, P.M. 1970, Astrophys. J., 159, 879.

Marsh, K.A. 1975, Astrophys. J., 201, 190.

Morton, D.C. \& Wright, A.E. 1978, Mon. Not. R. astr. Soc., 182, 47 P.

Olnon, F.M. 1975, Astr. Astrophys., 39, 217.

Panagia, N. \& Felli, M. 1975, Astr. Astrophys., 39, 41. 
Raymond, J.C., Cox, D.P. \& Smith, B.W. 1976, Astrophys. J., 204, 290. Schwartz, P.R. \& Spencer, J.H. 1977, Mon. Not. R. astr. Soc., 180, 297. Seaquist, E.R. 1976, Astrophys. J. (Lett)., 203, L35.

Seaquist, E.R. \& Gregory, P.C. 1973, Nature Phys. Sci., 245, 85.

Thomas, J.A., Robinson, G. \& Hyland, A.R. 1976, Mon. Not. R. astr. Soc., $174,711$.

Thorne, K.S. 1975, Orange Aid Preprint No. 421, California Institute of Technology.

Wendker, H.J., Baars, J.W.M. \& Altenhoff, W.J. 1973, Nature Phys. Sc1., 245,118 .

Wendker, H.J., Smith, L.F., Israel, F.P., Habing, H.J. \& Dickel, H.R. 1975, Astr. Astrophys., 42, 173.

Williams, P.M., Stewart, M.J., Beattie, D.H. \& Lee, T.J. 1977, IAU Circ. No. 3107.

Wood, F.B. 1977, Proc. IAU Colloquium No. 42, eds. R. Kippenhahn, J. Rahe, W. Strohmeier, p.639.

Woodsworth, A.W. \& Hughes, V.A. 1977, Astr. Astrophys., 58, 105. Woolf, N.J., Stein, W.A. \& Strittmatter, P.A. 1970, Astr. Astrophys., $\underline{9}, 252$.

Wright, A.E. \& Barlow, M.J. 1975, Mon. Not. R. astr. Soc., 170, 41.

\section{DISCUSSION FOLLOWING BARLOW}

Hearn: Cassinelli and Hartmann have shown that the infrared intensity of a star is given by $4 \pi R_{\lambda}^{2} B_{\lambda}$. At different wavelengths the radius of the star is determined by where the optical depth $\zeta_{\lambda} \simeq 1$ occurs. With ordinary hydrostatic models the density decreases so quickly that the radius is not significantly dependent on the wavelength and the infrared intensity is determined only by the variation of the Planck function.

The interpretation of the infrared data gives the density temperature structure. The interpretation of mass loss involves a further interpretation of the density structure.

Conversely the IR region, particularly in the range $10 \mu$ to $500 \mu$, will give information on the density-temperature of the stars in the region between the photosphere and corona.

Underhill: What are the temperatures in your IR emitting regions?

Barlow: For P Cygni itself, the temperature can safely be taken to be $20,000^{\circ} \mathrm{K}$. For some other stars we have taken somewhat higher values, of order $40,000^{\circ} \mathrm{K}$ as given by the example of $\zeta$ Pup.

Underhill: They do not seem to be as high as a few hundred thousand degrees?

Barlow: We cannot use the IR data to make a statement, one way or the other. Admittedly, the temperature adopted is important. The ratio data give the mass loss rates without much temperature dependence. 
Castor: Tony is right that from the modeling you find $\rho(r)$, not $\mathrm{v}(\mathrm{r})$. However, even with inhomogeneities it is true that $\overline{v(r)}=$ constant $/\left[r^{2} \frac{\rho(r)}{\rho(r)}\right.$ so as long as we realize that $\rho$ and $v$ are means, we can use the usual relationship.

Hearn: If the extended density structure obtained from the IR observations results from some other physical process, such as the wave pressure on turbulent pressure, the mass loss interpretation could be quite wrong. With the right sort of turbulent pressure, presumably one could explain the IR observations without any mass loss at all.

D. Van Blerkom: HD 50896 (WN5) was studied by Hartmann and Cassinelli, who suggested a region of decelerated flow in the envelope was necessary to account for the observed infrared flux distribution. Has any subsequent work been done on this star to confirm this unexpected velocity distribution?

Cassinelli: We could get a fit to the IR flux with a density falling as $1 / \mathrm{r}^{2}$, if the radius of the star is small $\sim 2.5 \mathrm{R}_{\odot}$. One can also get information about the density structure by looking at an eclipsing binary at IR wavelengths. Recently Lee Hartmann published results of his IR observations of V $444 \mathrm{Cyg}$, an eclipsing WR system. He found that the extended density distribution that we derived for HD 50896 gave an excellent fit to the IR light curve. 